\title{
A ubiquitous knowledgeable data representation model (UKRM) for three-dimensional geographic information systems (3D GIS)
}

\author{
ZHANG ShuQing ${ }^{1,2^{*}}$, ZHOU ChengHu ${ }^{2,3}$, ZHANG JunYan ${ }^{1} \&$ CHEN XiangCong ${ }^{1,2}$ \\ ${ }^{1}$ Northeast Institute of Geography and Agroecology, Chinese Academy of Sciences, Changchun 130102, China; \\ ${ }^{2}$ University of the Chinese Academy of Sciences, Beijing 100049, China; \\ ${ }^{3}$ State Key Laboratory of Resources and Environment Information System, Institute of Geographic Sciences and Natural Resources, \\ Chinese Academy of Sciences, Beijing 100101, China
}

Received April 10, 2015; accepted September 6, 2015; published online January 21, 2016

\begin{abstract}
In the face of complicated, diversified three-dimensional world, the existing 3D GIS data models suffer from certain issues such as data incompatibility, insufficiency in data representation and representation types, among others. It is often hard to meet the requirements of multiple application purposes (users) related to GIS spatial data management and data query and analysis, especially in the case of massive spatial objects. In this study, according to the habits of human thinking and recognition, discrete expressions (such as discrete curved surface $(D C S)$, and discrete body $(D B)$ ) were integrated and two novel representation types (including function structure and mapping structure) were put forward. A flexible and extensible ubiquitous knowledgeable data representation model (UKRM) was then constructed, in which structurally heterogeneous multiple expressions (including boundary representation (B-rep), constructive solid geometry (CSG), functional/parameter representation, etc.) were normalized. GIS's ability in representing the massive, complicated and diversified 3D world was thus greatly enhanced. In addition, data reuse was realized, and the bridge linking static GIS to dynamic GIS was built up. Primary experimental results illustrated that UKRM was overwhelmingly superior to the current data models (e.g. IFC, CityGML) in describing both regular and irregular spatial objects.
\end{abstract}

Keywords Discrete curved surface (DCS), Discrete body (DB), Discrete structure, Function structure, Mapping structure, "2D/3D" integrated representation, 3D GIS data model, UKRM model

Citation: Zhang S Q, Zhou C H, Zhang J Y, Chen X C. 2016. A ubiquitous knowledgeable data representation model (UKRM) for three-dimensional geographic information systems (3D GIS). Science China Earth Sciences, 59: 780-794, doi: 10.1007/s11430-015-5255-7

\section{Introduction}

The 2D geographic information systems (GIS), originated from computer-aided cartography in the 1960s, has gone deep into all walks of life, for example, land management, municipal administration, irrigation, fire services, transportation and urban planning, among others (Xiao et al., 2001). From the viewpoint of data structure, its spatial data is ob-

*Corresponding author (email: zhangshuqing@ neigae.ac.cn) tained and stored in such a way that function prototypes describing spatial objects' geometric shapes are discretized on the basis of differential theory. The theory and application of 2D GIS is now reaching maturity. The real world, however, is three-dimensional or even four-dimensional, for example, there exist not only 3D static objects like houses, buildings, geological bodies, but man-made or natural dynamic objects such as vehicles, ships, unmanned aerial vehicles, satellites, typhoon, landslides, etc. Consequently, it is inevitable to expand the 2D GIS into 3D GIS and temporal GIS (Yuan et al., 2011). Although current static, 
symbolic 2D GIS, and 3D GIS mainly stemming from the former, have made much progress in various aspects, particularly in geo-visualiza- tion, the confliction between the virtual space of traditional cartographic GIS and the real world space, as well as the cognitive space of human beings, is increasingly protruding (Zhu, 2014). The deficiencies of GIS are becoming even more obvious, especially with the advancement of spatial data acquisition, data storage and computation, as well as the diverse application and rising demand. It can hardly meet the demands of society for representing and analyzing the large-scale, high dynamic, diversified three-dimensional world.

The 3D GIS data model and data structure describes spatial objects and their relations in digital forms, and it is the basis of 3D GIS (Shi et al., 2003; Gong et al., 2004; Zhou et al., 2008). Bridging the gap between the virtual world and the reality, GIS data model is also regarded as the core of geographic information sciences (Reitsma, 2013). Spatial concept models involve the information of spatial objects about geometry, relation, topology, semantics, attributes, etc., of which, the core issue lies in the effective and efficient representation of geometry (Zlatanova and Tempfli, 2000). During the past two decades, the theory and technique of the 3D GIS data model and data structure have made considerable progress. Many 3D conceptual models have been proposed in the literature, but they still fall far behind the real demand. Compared with the $2 \mathrm{D}$, the three-dimensional world and its phenomena, under various human impacts, are much more complex and diversified; for example, manmade buildings may vary from culture to culture (Goodchild, 2009). The 3D GIS data model is not a mere increase of vertical $z$-coordinate in the traditional $2 \mathrm{D}$ GIS model, and cannot be realized through a simple expansion from lower into higher dimension; instead, the corresponding cognitive and thinking habits should be altered. But the current 3D GIS data models have the following serious shortcomings.

First, among existing data models, one category is to meet application requirements of a specific field or to consider certain GIS functions (Zlatanova et al., 2004). This category is called application-specific models (Zhou et al., 2008), which includes, for instance, 3D geo-database model used mainly for geological modeling (Mallet, 2002), Urban Data Model (UDM) for representing urban objects (Coors, 2003), semantics-based 3D dynamic hierarchical house property model for 3D cadastral management (Zhu and $\mathrm{Hu}$, 2008), Simplified Spatial Model (SSM) for web-oriented applications (Zlatanova and Tempfli, 2000), etc. But it is hard for an application-specific model to be used in different application fields. The other category, belonging to relatively general models, consists of models such as 3D Formal Data Structure (FDS) (Molenaar, 1991), Tetrahedron Network (TEN) (Pilouk, 1996), ISO 19107 Geographic Information-Spatial Schema (Herring, 2001), CityGML (Gröger et al., 2012), and so forth. To be universally suita- ble for geometric description, the simplest (or a simpler) primitive of each dimension is usually employed in these kinds of data models, e.g., triangle in 2-dimension and tetrahedron in 3-dimension. Theoretically, although any complex object can be approximated by these primitives, severe data redundancy may take place. One spatial object is usually expressed with a huge number of primitives (a large data set), let alone massive objects, which GIS often deals with. Besides, due to the fact that the geometric units of the current primitives are usually much smaller than those perceived by human beings, i.e. inconsistency between data storage and data analysis, accumulation of primitives is often required to represent a spatial entity that is in possess of semantical information. This would further bring about problems for 3D data management, data query, analysis and visualization (Zhang and Zhang, 2010). It is therefore imperative to develop a novel integrated GIS data model, with an aim to integrate different data models, represented with efficiency, having wide applicability, and to satisfy multiple GIS application requirements ( $\mathrm{Li}$ and $\mathrm{Li}, 1998$; $\mathrm{Lv}$ et al., 2013).

Second, the types of data representations are insufficient. In reality, many man-made features such as a circular plaza, a dome building, a parabolic cooling tower in a power plant etc., are perfectly described with theoretical functions. But all current data models belong to discrete data models that are comprised of discrete entities like nodes and triangles, thereby lacking functional representation (Breunig and Zlatanova, 2011). Such a discrete data model can result in massive volumes of data on one hand, for instance, the file sizes of CityGML are much larger than those of Building Information Model (BIM) which has functional, parametric representations (Berlo and Laat, 2011). On the other hand, accuracy errors can be produced due to the discretization of theoretical functions (e.g. discretizing a spherical function into triangles) (Kuttig, 2003). What's more, due to the lack of parametric/functional representation in GIS, a major problem of information lossless transformation exists between GIS and other software (like BIM and CAD).

Third, data reuse is not considered. In a community unit, many buildings might be completely equal to each other in terms of geometry, except for the difference in position (i.e. displacement) and orientation (i.e. attitude in 3D); similarly, the inner structures of each storey (or unit) in a building are usually the same too. In the process of architectural design, only one of the architectural drawings of those equally shaped buildings is made, while each of the rest of the buildings is simply marked with its position and orientation on a planar map. This is the concept of mapping used here. This paper will introduce the mapping concept into GIS, by just storing displacement and attitude, to reduce the cost of repetitive data modeling and storage, and improve data reuse and the efficiency of data production. It should be pointed out that the high cost of data generation and data updating is one of the major bottlenecks to the development 
and application of 3D GIS.

In general, despite the emergence of modern computational environments, existing 3D GIS data models are still inefficient in data modeling, data analysis and data generation, in the face of the complicated and diversified three-dimensional world. And the demands of GIS users for highly dynamic, massive objects, and multi-application, are not satisfied. An important reason is that, with primitives smaller than the basic geometric units of human beings' recognition and analysis, and with a unitary semantic hierarchy, the knowledge characteristics of the real world are hardly represented in current data models. In order to make innovation of 3D GIS data model, it should not only adopt the geometric knowledge of human beings, but draw on the experience of 3D mature software (e.g. CAD, 3DMax) (called ubiquitous knowledgeable), and more over, it should draw nutrients from other disciplines such as mathematics, computer science, engineering, etc. For example, as a general rule, architectural designers do not make design for each of the identical buildings repetitively, while GIS data model should also incorporate the concept of data reuse to reduce the cost of human resource as well as computer storage resource.

In this study, on the basis of cognitive granularities of human beings, existing 3D data models (e.g. boundary representation (B-rep), constructive solid geometry (CSG), etc.) are systematically expanded to achieve an efficient "discrete structure", in which parameterization is allowed; in addition, two novel representation types: function structure and mapping structure are put forward. Each of the structure types may contain several sub-classes of structures, for instance, discrete structure consists of sub-classes like discrete unit $(D U)$, set operation, etc. A knowledgeable representation data model (called UKRM, ubiquitous knowledgeable representation model) is then constructed under a unified framework, in which the three structure types: discrete, function, and mapping structure are well integrated. UKRM exhibits favourable properties in terms of flexibility, extensibility and maintainability; according to application demands, new structures can be conveniently defined and maintained by a GIS user. This enables us to integrate humans' knowledge and awareness of the objective world, so that the complex and diversified 3D world can be flexibly and efficiently described. It should be noted that the 3D model serves as the bridge for connecting 2D and dynamic GIS: from geometric viewpoint, $2 \mathrm{D}$ is the $2 \mathrm{D}$ projection of 3D while dynamic GIS is " $3 \mathrm{D}+$ instant attitude of object". With this logical relationship in mind, an integrated data modeling of 2D/3D and association between 3D and dynamic GIS are achieved, thanks to the definition of $2 \mathrm{D}$ " $R I N G$ " and the incorporation of "mapping structure". This paper puts emphasis on expounding the knowledgeable presentation data model (UKRM). Despite the ability of UKRM in meeting the requirements of multi-purpose applications, only modeling applications are im- plemented and verified through comparing with CityGML 2.0 and IFC $2 \times 4$, due to the length limitation of this paper.

\section{Basic concepts and definitions}

Writing conventions: to get a clear presentation, an element of a set is stipulated as follows: a basic element is denoted with a word in lowercase, e.g. shapeType; a set is denoted with a word in uppercase, e.g. $B O X$ (the minimum bounding box of a regular tetrahedron); while a set's set, called family set, is denoted with a word in uppercase with square brackets, e.g. $[B O X]$ (i.e. the family set of $B O X$ ).

(1) $2 \mathrm{D}$ and $3 \mathrm{D}$ spatial point:

$$
\begin{aligned}
& P 2 D=\{x, y\}, \\
& P=\{x, y, z\} .
\end{aligned}
$$

(2) Operator op: including symbols of set operations (e.g. Union " $\cup$ ", Intersect " $\cap$ ", and Difference " "), brackets "(", ")", and so on.

(3) Facet and sub-surface. A facet is a bounded planar polygon (or triangle) in 3D space; sub-surface refers to a facet set constructed by several facets, or a bounded curve surface defined by a function.

(4) Sub-object. If a spatial object is formed by a number of smaller objects, these smaller objects are then called sub-objects. In the process of data modeling, sub-objects can be partitioned based on macro-geometrical or semantic properties.

(5) Composite object/sub-object. Four basic shape types (denoted as shapeType), namely, point, line, surface and solid are defined in this paper. If an object (or sub-object) consists of more than one basic shape types, it is then called a composite object (or sub-object).

(6) Auxiliary object/sub-object. For the convenience of GIS data representation, data analysis and so on, an object (or sub-object) that is not in existence may be artificially generated. Such an object (or sub-object) is then called auxiliary object (or sub-object). For example, in Figure 1, $O b j=S u b O b j_{2} / S u b O b j_{1}$, where $O b j$ is the real object while

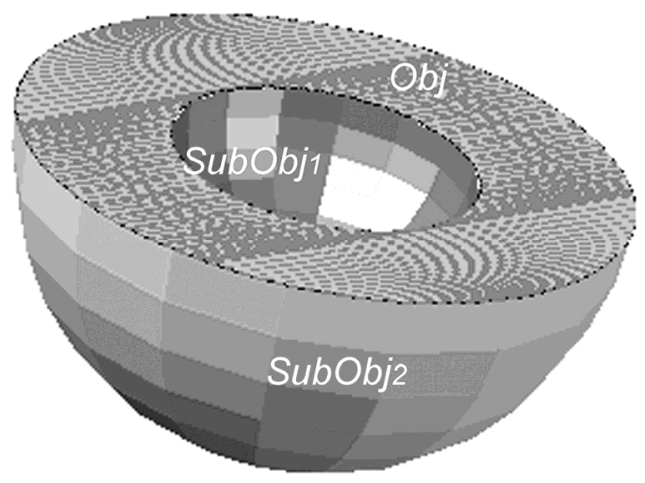

Figure $1 O b j$, the spatial object, is modeled through set difference operation between auxiliary sub-objects $\left(\mathrm{SubObj}_{2}, \mathrm{SubObj}_{1}\right)$. 
$\mathrm{SubObj}_{2}$ and $\mathrm{SubObj}_{1}$ are two auxiliary objects (or sub-object). "/" refers to set difference operation.

(7) RING. RING refers to the bounded boundary of the projection (on 2D plane) of an object (or sub-object), which is only applicable to a surface or solid object (or subobject), or a composite object (or sub-object) with surface or solid component(s). RING can be a bounded curved line defined by a function. If constructed with discrete points, $R I N G$ is then expressed as: $[R I N G]=\{n p,[P 2 D]\}$, where $n p$ is the number of points, $[P 2 D]$ is the family set of the $2 \mathrm{D}$ coordinates of points.

(8) Mapping, ATTITUDE and (OFFSET or $D I S P L A C E M E N T)$. Suppose $(\alpha, \beta, \gamma)$ is the respective angle between the three coordinate axis of the local coordinate system of a moving object and those of the global coordinate system (Figure 2). The attitude matrix $\left(\left\{a_{i j}\right\} ; i, j \in[1,3]\right)$ formed by the three angles are called ATTITUDE, while OFFSET (or DISPLACEMENT) is the coordinate difference between the origins of the two coordinate systems. By making use of an existing object (or sub-object), called an elementary object (or sub-object), a new object (or subobject), called mapping object (or sub-object), can be automatically generated by employing transformation of ATTITUDE and/or OFFSET. Here, a spatial object (or sub-object) of any shape type (point, line, surface, solid or composite) can serve as an elementary object (or subobject).

\section{Ubiquitous knowledgeable representation model (UKRM)}

Three types of structures, namely discrete structure, function structure and mapping structure, are included in the UKRM model (see Section 3.2 for details), of which, discrete structure ranks the top place. While as the major form of the discrete structure, discrete unit $(D U)$ structure (i.e. basic unit of discrete data) plays an important role in data representation, thus being emphasized first.

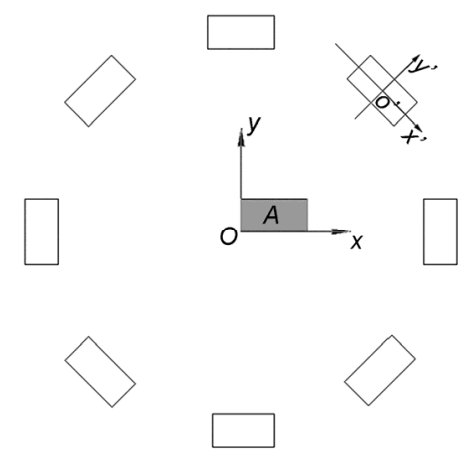

Figure 2 2D sketch map of mapping. The grey rectangle is the elementary object while other rectangles are mapping objects. Oxyz is the grobal coordinate system transformed to elementary object $A, O^{\prime} x^{\prime} y^{\prime} z^{\prime}$ is the local coodinate system of mapping object $A^{\prime}$.

\subsection{Discrete unit $(D U)$}

According to the method of data organization, discrete unit $(D U)$ can be divided into two categories: discrete curved surface $(D C S)$ (an explicit structure) and discrete body $(D B)$ (a semi-implicit structure). Here, trip type (denoted as tripType) is used to refer to the expression form of a particular $D C S$ or $D B$ structure.

\subsubsection{Discrete curved surface (DCS)}

Discrete curved surface (DCS) describes a curved surface (plane is regarded as a special form of curved surface) using discrete data, in which the graph of points, facets (or double-ruled surface) and their relations are directly presented. Different trip types of DCS are defined as follows.

(1) Triangle fan. The conventional triangle fan: the first point and each two neighboring points in a data chain form a triangle. In Figure 3(a), for instance, points are stored in order: $\left\{P_{1}, P_{2}, P_{3}, P_{4}, P_{5}, P_{6}, P_{7}\right\}$ (i.e. $\left.[P]\right)$. The number of points $(n p)$ and the number of triangles $(n s)$ has the following relations,

$$
n p=n s+2 .
$$

A triangle fan can be represented as:

$$
D C S=\{\text { tripType }, n p,[P]\},
$$

where tripType is "triangle fan", $[P]$ is the family set of points.

(2) Triangle strip. The conventional triangle strip: every three neighboring vertices in a data chain constitute a triangle (Figure 3b). The amount of storage space of a triangle strip is the same as that of a triangle fan, and the relation between $n p$ and $n s$ is the same as eq. (2). A triangle strip can also be represented with eq. (3), in which the tripType $=$ "triangle strip".

(3) Quadrilateral strip. Points are stored in pairs in a data chain, and one quadrilateral is then defined with each pair of points in combination with the previous pair. In Figure 3(c), for example, the data chain is read in order $\left\{\left(P_{1}, P_{2}\right)\right.$; $\left.\left(P_{3}, P_{4}\right) ;\left(P_{5}, P_{6}\right) ;\left(P_{7}, P_{8}\right)\right\}$, where two consecutive groups (pairs) form a quadrilateral. This trip type is also expressed by eq. (3), in which the tripType = "quadrilateral strip", and the relation between $n p$ (the number of points) and $n s$ (the number of facets) is:

$$
n p=2 \cdot n s+2 .
$$

Similarly, a double ruled surface strip (Figure 3d) can be designed to represent certain spatial objects or phenomena (e.g. terrain, geological fault, and so on).

(4) Multi-point consecutive trip (MCT). It represents, once for all, the facets that have the same number of edges and are semantically identical, but may separate from each other as shown by Figure 3e. This trip type takes the form of: 


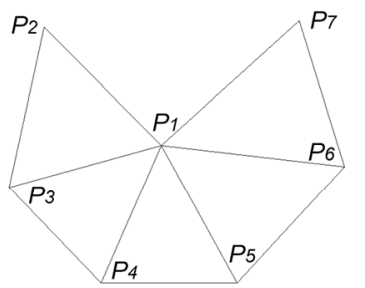

(a)

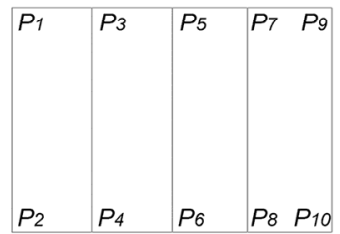

(c)

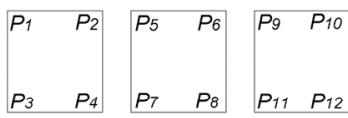

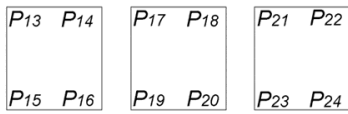

(e)

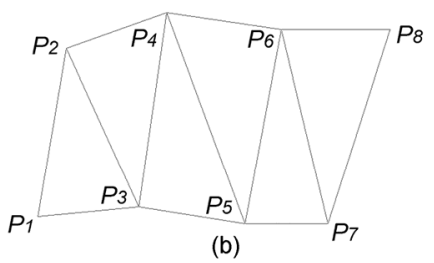

(b)
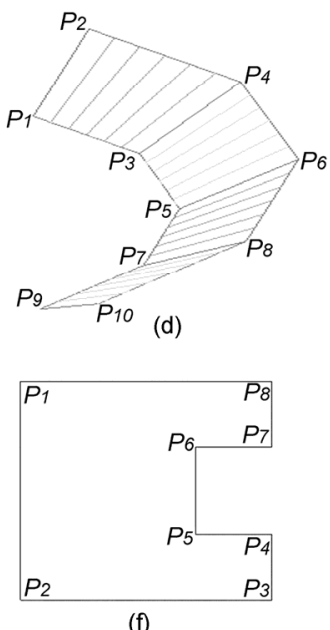

(f)
Figure 3 DCS with different trip types. (a) Triangle fan, (b) triangle strip, (c) quadrilateral strip, (d) double ruled surface strip, (e) multi-point consecutive trip (MCT), and (f) multi-point independent trip (MIT).

$$
D C S=\{\text { tripType, } n s, n p,[P]\},
$$

where $[P]=\left\{\left\{P_{1,1}, P_{1,2}, \ldots, P_{1, n p}\right\}, \ldots,\left\{P_{i, 1}, P_{i, 2}, \ldots, P_{i, j}, \ldots\right.\right.$, $\left.\left.P_{i, n p}\right\}, \ldots,\left\{P_{n s, 1}, P_{n s, 2}, \ldots, P_{n s, n p}\right\}\right\}(1 \leqslant i \leqslant n s, 1 \leqslant j \leqslant n p), n s$ is the number of facets, $n p$ is the number of edges of any facet, and tripType $=$ " $M C T$ ". Figure $3 \mathrm{e}$ illustrates a $M C T$ composed of 6 squares. Windows, doors, or the like, in a building might be collectively expressed with this trip type.

(5) Multi-point independent trip (MIT). Facets that may differ in the number of points from each other are depicted by MIT as follows:

$$
D C S=\{\text { tripType },\{n s, N S P\}, n p,[P]\},
$$

where tripType $=$ "MIT", $n s$ is the number of facets, NSP is a data set whose elements respectively describe the number of each facet's points, $n p$ is the total number of points of all facets, and $[P]$ refers to the set family of points. With less data, a building's up or bottom surface made up of facet(s) can be efficiently represented by this trip type. Figure $3 f$ delineates a multi-point independent trip (i.e. an irregular polygon), where $n s=1, N S P=\{8\}, n p=8,[P]=\left\{P_{1}, P_{2}, \ldots\right.$, $\left.P_{8}\right\}$.

Besides the above-mentioned DCS (i.e. discrete surface primitives), other forms of $D C S$ could also be designed to represent a surface or sub-surface. Of course, a solid object (or sub-object) can also be described with its surface closely bounded by DCSs (i.e. B-rep).

\subsubsection{Discrete body $(D B)$}

Discrete body $(D B)$ is a three-dimensional geometric body (solid) bounded by sub-surface(s). A trip type (tripType) of $D B$ structure describes implicitly the entire surface of a particular body (solid), and moreover, the shape of the solid is implicitly delineated too. The definition of a $D B$ structure adopts discrete data (e.g. vertices) and parameters (due to the high efficiency in data description, parameters should be used as much as possible). $D B$ structures possess favourable properties such as structural compactness, less data, and so on. In fact, this type of structure has already been used in real applications, for instance, only four vertices are required to represent a tetrahedron, indicating implicitly that any three of the four vertices forms a facet (i.e. a triangle). Broadly, there are two types of $D B$ structures: vertical discrete body $(D B)$ trip and oblique body trip. Here, Vertical $D B$ trip has two important properties: (1) a solid is expressed with a $2 \mathrm{D} R I N G$ formed by vertices (i.e. $[P 2 D]$ ), and a family set of other parameters (denoted as [OTHERS]); and (2) all images of the vertices mapped on Oxy coordinate plane lie within the 2D RING. Suppose the number of vertices on a RING is $n p$, then the general model of vertical $D B$ trip is expressed as:

$$
[D B]=\{\text { tripType }, n p,[P 2 D],[\text { OTHERS }]\},
$$

where tripType refers to the trip type.

Typical vertical $D B$ trips include right prism, house ridge, wedge, regular prismoid, etc. But due to the length limitation of this paper, only right prism, house ridge and wedge are taken as examples, to illustrate the way of defining a specific $D B$ structure here.

(1) Right prism ([PRISM]): the set expression of a right prism is as follows:

$$
[P R I S M]=\left\{\text { tripType }, n p,\left\{[P 2 D], z_{0}, h\right\}\right\},
$$

where tripType = "right prism"; $[P 2 D]$ - the $2 \mathrm{D}$ RING of the prism formed by the family set of $2 \mathrm{D}$ footprints of the prism-is explicitly presented in the model, $n p$ is the number of the footprints, $z_{0}$ is the elevation of the right prism's bottom facet, and $h$ is the absolute height of the prism itself. Meanwhile, the bottom face (facet), the up face (facet), and side faces (facets) of the prism are given implicitly.

(2) House ridge ([RIDGE]): house ridge is a common building structure often appearing in the top of a house, which is a special form of polyhedron (Figure 4a), whose expression is as follows:

$[R I D G E]=\left\{\right.$ "houseridge", $n p,[P 2 D], z_{B}, h$, offset $\}$,

where $[P 2 D]$ is the $2 \mathrm{D}$ coordinate family set of the vertices of the ridge's bottom facet, $n p$ is the number of the vertices in $[P 2 D], z_{B}$ is the elevation of the ridge's bottom facet, $h$ is the height of the ridge, offset is the distance between the end of the ridge line and the boundary of the bottom facet (Figure 4b). 
(3) Wedge ([WEDGE]): eq. (8) is also used as the set expression of a wedge (Figure 5), in which the tripType ="wedge".

\subsection{Structure types}

The structure types (denoted as structureType) defined in this paper include three categories: discrete structure, function structure and mapping structure, each of which may further comprise several sub-classes of structures. A detailed description about each structure type is given hereafter.

\subsubsection{Discrete structure.}

In discrete structure, an object (or sub-object) might be composed of a number of smaller objects, or it might be derived from set operation(s) on a set of sub-objects. Three major sub-classes of structure types including discrete unit structure, set operation structure, and discrete object structure, are elaborated here:

(1) Discrete unit structure ([DUS]). An object (or sub-object) is represented with discrete unit $(D U)$ structure, which is modeled as follows,

$$
\begin{gathered}
{[D U S]=\{n d u,[D U]\},} \\
{[D U S]=\{\{n p,[P]\},\{n d u,[D U]\}\},}
\end{gathered}
$$

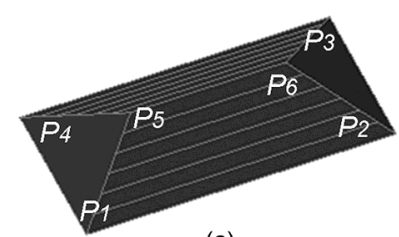

(a)

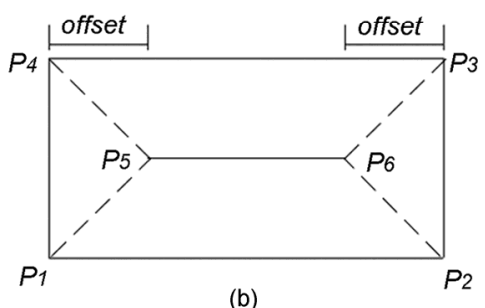

Figure 4 Three-dimensional graph of ridge (a), and its 2D projection map (b). $P_{5}, P_{6}$ is the ridge line; $P_{1} P_{2} P_{3} P_{4}$, the bottom facet; offset, the distance between $P_{5}$ and $P_{1}, P_{4}$ (or between $P_{6}$ and $P_{2}, P_{3}$ ).

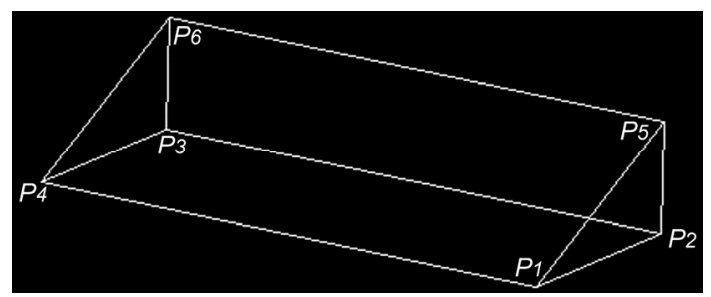

Figure 5 3D graph of wedge. where eq. (10a) and (10b) is non-topological and topological equation of $D U$ structure respectively; $[D U]$ can be either $[D C S]$ (family set of discrete curved surface $(D C S)$ or family set of discrete body $(D B)$ ); for topological structure, $[D U]$ just stores the references of points; $n d u$ is the corresponding number of $D C S$ or $D B$; $n p$ is the number of points, and $[P]$ is the family set of points in the topological structure.

(2) Set operation structure ([SOP] for brief). If an object (or sub-object) is generated through set operation on existing sub-objects, then it is defined as set operation structure, whose model is as follows:

$$
[S O P]=\{n S u b O b j N u m, \text { nop }, S U B N U M, O P\},
$$

where $n$ SubObjNum is the number of sub-objects (including auxiliary sub-objects) being referenced (indexed); nop is the number of operators; $S U B N U M$ is the set of sequential numbers of the sub-objects for reference; $O P$ is the set of postorder operators.

(3) Discrete object structure ([DOS]). If an object (or sub-object) is composed of more than one sub-objects (including auxiliary sub-objects), then the structure is called discrete object structure, which is expressed as follows:

$$
\begin{gathered}
{[D O S]=\{n s o,[B O X],[S O]\},} \\
{[D O S]=\{n s o,[S O]\},}
\end{gathered}
$$

where, eq. (12a) refers to discrete object structure with $B O X$ (the bounding box) for each sub-object, which suits the representation of an object, whose sub-objects have a relatively large number of points, whereas eq. (12b) is the discrete object structure without $B O X$ for sub-objects with fewer points; nso is the number of sub-objects, and [SO] is the family set of sub-objects; For set operation, the total sub-objects belonging to an object in discrete object structure include the sub-objects participating the computation of set operation and those derived from the set operations. For example, in Figure 1, the object is represented as $O b j=$ $\mathrm{SubObj}_{2} / \mathrm{SubObj}_{1}$, where only $\mathrm{Obj}$ belongs to the real object, whereas $\mathrm{SubObj}_{2}$ and $\mathrm{SubObj} j_{1}$ (two auxiliary sub-objects) do not, in this case $n s o=3$ (rather than 1). Without loss of generality, nso in eq. (12) stands for the total number of sub-objects in the structure data. In default, union set operation(s) are employed among sub-objects in a discrete object structure. Note: when building a sub-object with subobjects, discrete object structure (eq. (12)) is still used.

\subsubsection{Function structure ([FS])}

Function structure represents an object or sub-object with a function, whose model is as follows:

$$
[F S]=\{\text { functionName, PARAMETER }\},
$$

where functionName refers to the name of the function; PARAMETER is the set of parameters, in which variables might also be included. 
A variety of spatial objects or sub-objects (e.g. sphere, cone, hyperboloid, etc.) that have regular geometric shapes can all be described by functions. The definition of each function should provide the corresponding variables, parameters, as well as operators. Here, the range of variables and parameters is beyond that in mathematics (e.g. spherical center and radius of a sphere), instead, a discrete unit (DCS or $D B$ ) (i.e. geometric primitive of discrete data) can also be regarded as a parameter to define a function. As an illustration, this paper will put forward two functions: one is about an omidirectional spherical segment, in which variables, parameters and operators are included; the other one describes planar windows (or doors) distributed in the same pattern on different floors by incorporating a $D C S$ as a parameter.

(1) Spherical segment ([SSEGMENT]).

PARAMETER $=\left\{\left\{P_{0}, R, \alpha, \beta, S\right\},\{\geqslant, \leqslant\}\right\}$,

$[$ SSEGMENT $]=\left\{\right.$ "spherical segment", $\left\{\left\{P_{0}, R, \alpha, \beta, S\right\}\right.$, $\left.\left\{o p_{1}, o p_{2}\right\}\right\}$.

Suppose the plane equation of spherical segment (occurrence) plane is:

$F=x \cos \alpha \sin \beta-y \sin \alpha \cos \beta+z \cos \alpha \quad$ (Zhang and

Zhang, 2010), where $\alpha$ is the inclination angle, i.e. the angle between the norm of the spherical segment plane and $z$-axis; $\beta$ is the attitude angle, i.e. the angle between the projection line of the spherical segment facet and $x$-axis (Figure 6, where $A B C D$ can be regarded as the spherical segment plane), $R$ and $P_{0}$ is the radius and the origin of the sphere respectively; $S$ and $F$ is respectively the distance between the spherical segment plane and the center of the sphere, and that between the spherical segment plane and the origin of the global coordinate system. Two operators $\left(o p_{1}, o p_{2}\right)$

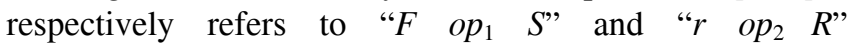
$\left(r=\sqrt{x^{2}+y^{2}+z^{2}}\right)$. The value of $o p_{1}$ and $o p_{2}$ can be any one of following operator symbols: ">”, “<”, “ $\geqslant$, “ $\leqslant$, "=" and " $\neq$ ". If the norm of the spherical segment plane is vertical upward (i.e. the plane is horizontal), then $\alpha=0, F \geqslant$ $S, r \leqslant R$. The function designed in such a way can describe a spherical segment at any size and in all directions. For the sake of the length limitation of this study, the detailed deduction process of the function is not presented.

(1) Window or door loop function ([WDL]).

$$
\begin{gathered}
{[W D L]=\{\text { "window or door looping", }} \\
\left.S N, h_{0}, n f, h,[D C S]\right\}, \\
{[W D L]=\{\text { "window or door looping", }} \\
\left.S N, h_{0}, n f, h,\{\text { "MCT", ns, np },[P]\}\right\},
\end{gathered}
$$

where, "window or door looping" is the function name, $S N$ (a POINT data type) refers to the unit normal of the planar surface (i.e. the plane coplanar with the windows or doors), $h_{0}$ is the elevation of the windows or doors of the floor to start (called start floor), $n f$ is the number of the floors, and $h$

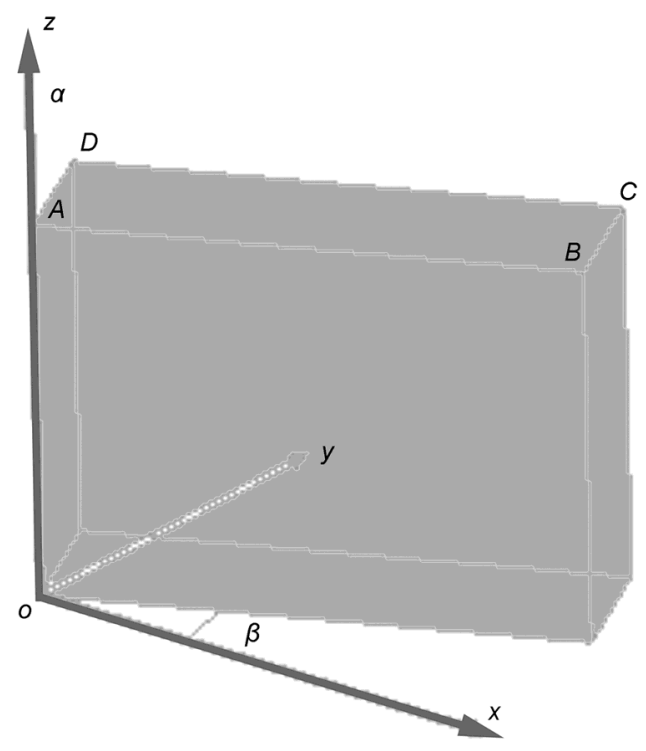

Figure 6 Sketch map of a facet's occurrence. Facet $A B C D$ is horizontal, $\alpha$ is the inclination angle between the norm of the facet and $z$-axis, in this case $\alpha=0 ; \beta$ is the attitude angle.

is the floor height. $[D C S]$ in eq. (15a) is the structure data of the start floor's windows (or doors), and it is expressed in multi-point consecutive trip (MCT) in eq. (15b).

In addition to commonly used variables and parameters, operator symbols, as well as $D C S$ or $D B$, mapping structure (see Section 3.2.3) can also serve as a parameter to define a function.

\subsubsection{Mapping structure ([MPS])}

Mapping structure represents a new object (or sub-object) based on an existing object (or sub-object) by endowing a new attitude and/or coordinate offset, expressed as follows:

$$
[M P S]=\{\text { OFFSET }, \text { ATTITUDE },[\text { VARIABLE }]\},
$$

where OFFSET (a POINT data type) is the offset of the local coordinate system's origin, ATTITUDE is the attitude of the local coordinate system with respect to the global coordinate system, i.e. the $\left\{a_{i j}\right\} \quad(i, j \in[1,3])$ attitude matrix; [VARIABLE] is the family set of variables: if it is INDEX, then the [MPS] is called index mapping structure; if it is a function i.e. $[F S]$, then the $[M P S]$ is called function mapping structure. Besides, [VARIABLE] can be used uniquely or in combination with OFFSET to form structures like index structure, offset structure, etc. Detailed descriptions about these structure types will be given hereafter:

(1) Index structure (INDEX). Without coordinate transformation, an object (or sub-object) is generated by directly referencing to an existing object (or sub-object). Such a structure is called index structure, which is modeled as follows:

$$
\text { INDEX }=\{\text { dataOffset, dataCL, connectString }\},
$$

where dataOffset, dataCL and connectString are respec- 
tively the data offset, the content length and the file path of the data being indexed. Whether the RING of the object (sub-object) is referenced depends on the contents and data length.

(2) Index mapping structure ([IMS]). A new mapping object (or sub-object) is generated, by employing data transformation of ATTITUDE and OFFSET on an existing object (or sub-object). This structure type is defined as index mapping structure, whose model is as follows:

$$
[I M S]=\{\text { OFFSET }, \text { ATTITUDE, INDEX }\},
$$

where INDEX is expressed by eq. (17). Of course, users can either directly store the data on the basis of eq. (18), or store the instantiated data after data transformation based on eq. (19). Suppose [ $\left.P^{\prime}\right]$ is the family set of the points to be indexed, and $[P]$ is the family set of the points of the mapping object (or sub-object) after data transformation, which can be calculated as follows:

$$
\begin{gathered}
{[P]=\text { OFFSET }+ \text { ALTITUDE }\left[P^{\prime}\right],} \\
\left(\begin{array}{l}
x \\
y \\
z
\end{array}\right)=\left(\begin{array}{l}
x_{0} \\
y_{0} \\
z_{0}
\end{array}\right)+\left\{\begin{array}{lll}
a_{11} & a_{12} & a_{13} \\
a_{21} & a_{22} & a_{23} \\
a_{31} & a_{32} & a_{33}
\end{array}\right\} \cdot\left\{\begin{array}{l}
x^{\prime} \\
y^{\prime} \\
z^{\prime}
\end{array}\right\},
\end{gathered}
$$

where $\left\{a_{i j}\right\}(i, j \in[1,3])$ is the attitude matrix of the mapping object (or sub-object), $\left(x_{0}, y_{0}, z_{0}\right)$ is the corresponding local coordinate system's origin, i.e. OFFSET (Figure 2).

(3) Offset mapping structure ([OMS]). A new object (or sub-object) is formed by employing OFFSET coordinate transformation on existing object (or sub-object), expressed as follows:

$$
[O M S]=\{O F F S E T, I N D E X\} .
$$

(4) Function mapping structure ([FMS]). it is to generate a new object (or sub-object) through coordinate transformation in terms of ATTITUDE and OFFSET on a functional object (or sub-object) in existence, which is modeled as:

$$
[F M S]=\{\text { OFFSET }, \text { ATTITUDE, }[F S]\},
$$

where $[F S]$ refers to any one of the functions defined by function structure. This structure amounts to the transforming and indexing on an existing functional object (or sub-object).

\subsection{UKRM model of object and sub-object}

The structure types involved in UKRM model are described in the above section. With these structures, the model of object (or sub-object) is then formulated. Hereinto, the object's model contains both data header (denoted as [HEAD]) and graph data (denoted as $[D]$ ) while the sub-object's model is expressed just by graph data $([D])$ :

$[H E A D]=\{$ objectNumber, shapeType, BOX $\}$,

$$
\begin{gathered}
{[D]=\{\text { structureType, }[\text { SDATA }], n r,[\text { RING }]\},} \\
{[\text { OBJ }]=\{[\text { HEAD }],[D]\},} \\
{[S O]=[D]=\{\text { structureType, }[\text { SDATA }],[\text { SA }], n r,[\text { RING }]\} .}
\end{gathered}
$$

Eq. (21a) formulates the data header ([HEAD]), in which objectNumber (the sequential number of the object), shapeType (the shape type: point, line, surface, body, or composite), and $B O X$ (the bounding box) are included; eq. (21b) describes the graph data $([H E A D])$, which consists of structureType (the structure type: DU structure, function structure, mapping structure, etc), [SDATA] (the structure data without including $R I N G$ ), $n r$ (the number of RINGs), and $R I N G$ (the 2D RING). Eq. (22c) presents the model of object $([O B J])$, while eq. (22d) describes the model of sub-object ([SO]); data header is assigned only to an object, rather than to a sub-object; for the convenience of mapping structure, structure type (structureType) is stored outside of structure data [SDATA]. [SA] refers to the family set of surface attributes (e.g. inner/outer property of a surface). Note: $[S A]$ is only applicable to a surface object (or sub-object), while for other shape types, [SA] is an empty set (i.e. [SA] $=\varphi)$.

Figure 7 is the UML graph of UKRM model. From eq. (22) and Figure 7, it can be seen that discrete structure, function structure and mapping structure can all be utilized for the description of an object or sub-object, that is, object (or sub-object) can be represented with any structure types. Hereinto, an object can be constructed with an arbitrary number (say $n$ ) of sub-objects, and it allows various structure types to be used jointly, thus greatly enhancing the flexibility of spatial object representation. When forming an object or sub-object with several sub-objects, discrete object structure is applied. Of course, an object can also be directly generated with $D U$ structure (i.e. DCSs or DBs) or function or mapping structure, which is equivalent to the case of an object having just one sub-object. For example, for a solid object (or sub-object), it can be either expressed with closely bounded $D C S(\mathrm{~s})$, or modeled with discrete body $(D B)$, or described with function or mapping structure. Here, we stipulate that set difference operation is only applicable to sub-objects constructed with function structure, as illustrated by Figure 1. Moreover, to limit the depth of the "object-structure" relationship tree, a convention is made in this paper that only a solid object (or sub-object), or a composite object (or sub-object) having solid component(s), is allowed to contain sub-objects further.

\section{Application of UKRM model}

\subsection{Steps of data modeling}

The data generation process can be sketched as in three steps:

(1) Step 1. Determine whether the object is expressed 


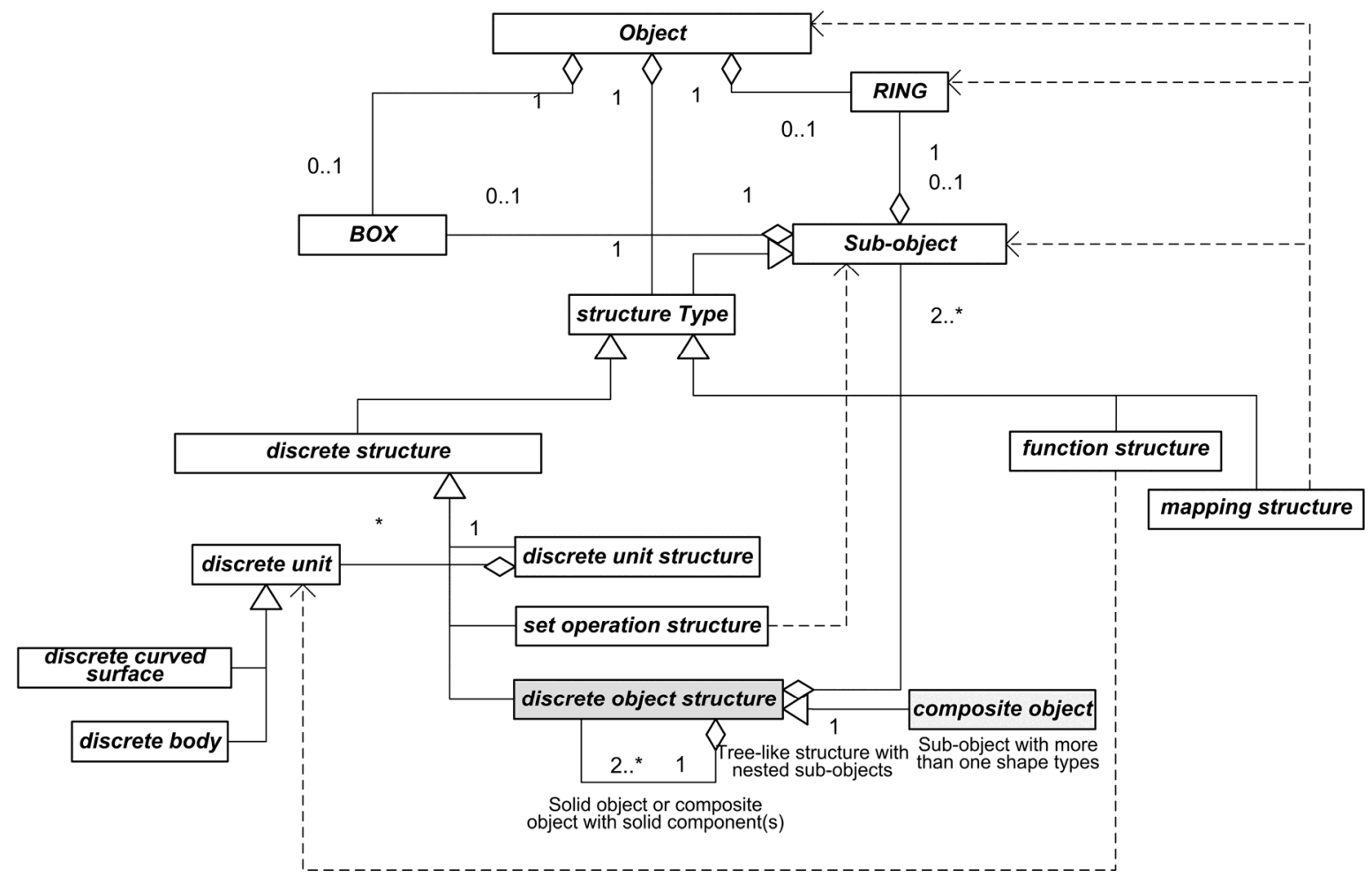

Figure 7 UML graph of the UKRM model.

with structures directly, if it is (the object just has one subobject), then go to Step 2A; otherwise (the object has several sub-objects), determine the number $(n)$ of sub-objects first, then go to Step 2B;

(2) Step 2A. Determine the object's structure type, $D U$ (DCS or DB), function structure or mapping structure, and construct the object based on the chosen structure type; stop the modeling process and return;

(3) Step 2B. Loop from 1 to $n$, determine the structure type of each sub-object, and generate the corresponding data of the sub-object;

(4) Step 3. Synthesize the structure data of the object using the sub-objects, based on discrete object structure, and build the model of the object (eq. (22)); return.

\subsection{Examples of application:}

Three examples: ore body modeling using DCS structure; simple building modeling using $D B$, function and mapping structure; and complex building modeling using $D C S, D B$, and function, as well as mapping structure, are presented to demonstrate UKRM model's adaptability in describing different types of spatial entities.

\subsubsection{Example 1}

Ore body modeling. Figure 8 shows an ore body depicted as a sub-object (without RING) with three DCSs: two triangle fans $\left(\left[D C S_{1}\right]\right.$ and $\left.\left[D C S_{3}\right]\right)$, and one triangle strip $\left(\left[D C S_{2}\right]\right)$. The modeling process of the sub-object based on non-

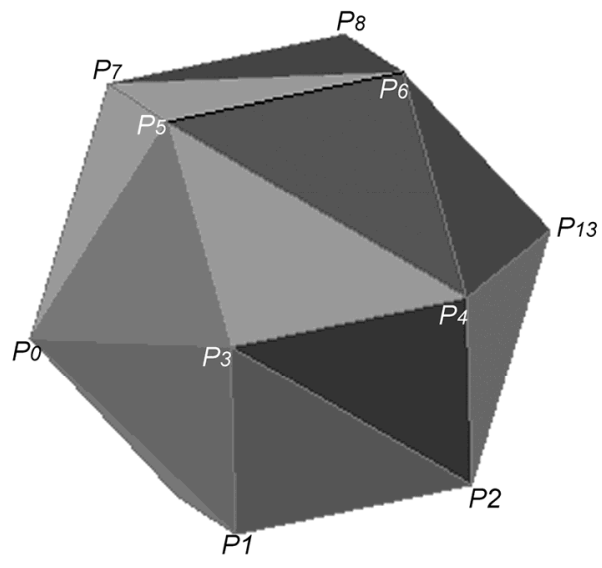

Figure 8 Ore body expressed based on DCS.

topological DCS structure (eq. (10a)) is presented first as follows.

(1) Step 1: describe the non-topological DCS structure data

$\left[D C S_{1}\right]=\{$ "triangle fan", $8,[P]\}=\left\{\right.$ "triangle fan", $8, P_{0}$, $\left.P_{1}, P_{3}, P_{5}, P_{7}, P_{9}, P_{11}, P_{1}\right\} ;\left[D C S_{2}\right]=\{$ "triangle strip", 14, $[P]\}=\left\{\right.$ "triangle strip", $14, P_{1}, P_{2}, P_{3}, P_{4}, P_{5}, P_{6}, P_{7}, P_{8}, P_{9}$, $\left.P_{10}, \quad P_{11}, \quad P_{12}, \quad P_{1}, \quad P_{2}\right\} ; \quad\left[D C S_{3}\right]=\{$ "triangle fan”, 8 , $[P]\}=\left\{\right.$ “triangle fan", $\left.8, P_{13}, P_{2}, P_{4}, P_{6}, P_{8}, P_{10}, P_{12}, P_{2}\right\}$

(2) Step 2: define the non-topological sub-object model ([SO]) of the ore body (structureType="non-topological $\left.D C S^{\prime \prime}\right)$

$[S O]=\left\{\right.$ “non-topological DCS", $\left\{3,\left\{\left[D C S_{1}\right],\left[D C S_{2}\right]\right.\right.$, 
$\left.\left.\left.\left[D^{2} S_{3}\right]\right\}\right\}\right\}=\{$ “non-topological DCS", $\{3,\{\{$ “triangle fan", 8, $\left.P_{0}, P_{1}, P_{3}, P_{5}, P_{7}, P_{9}, P_{11}, P_{1}\right\},\left\{\right.$ "triangle strip", 14, $P_{1}$, $\left.P_{2}, P_{3}, P_{4}, P_{5}, P_{6}, P_{7}, P_{8}, P_{9}, P_{10}, P_{11}, P_{12}, P_{1}, P_{2}\right\}$, \{ “triangle fan", 8, $\left.\left.P_{13}, P_{2}, P_{4}, P_{6}, P_{8}, P_{10}, P_{12}, P_{2}\right\}\right\}$ \}.

Moreover, the ore body can be expressed as a sub-object using topological DCS structure, based on eq. (10b) and (22d) as follows:

$[S O]=\{$ structureType, $\{\{n p,[P]\},\{n d u,[D C S]\}\}\}$, where structureType $=$ "topological DCS", $n p$ (the number of vertices $)=14,[P]$ (the family set of vertices $)=\left\{P_{0}, P_{1}, \ldots\right.$, $\left.P_{13}\right\}, n d u$ (the number of $D C S s$ ) $=3,[D C S]$ (the family set of $D C S \mathrm{~s}$ ). Suppose the sequential number of vertices $P_{0}$, $P_{1}, \ldots, P_{13}$ is $0,1, \ldots, 13$ respectively; each of three $D C S \mathrm{~s}$ is described by the DCS structure's trip type and the set of its vertices' sequential numbers, e.g., $\left[D C S_{1}\right]=\{$ "triangle fan", $8,0,1,3,5,7,9,11,1\}$. The topological structure of the sub-object ([SO]) is then expressed as:

$[S O]=\left\{\right.$ "topological DCS", $\left\{14,\left\{P_{0}, P_{1}, \ldots, P_{13}\right\},\{3\right.$, $\{\{$ "triangle fan”, 8,0, 1, 3, 5, 7, 9, 11,1\}, \{ "triangle strip", 14, 1, 2, 3, 4, 5, 6, 7, 8, 9, 10, 11, 12, 1, 2\}, \{ "triangle fan", $8,13,2,4,6,8,10,12,2\}\}\}$

\subsubsection{Example 2}

Representing a building having spherical segments. Suppose a building consists of three sub-objects, namely, one main building $(M B)$, and two identical spherical segments (SS1 and SS2) lying on the top of the main block (Figure 9). The building is expressed as a sub-object by synthesizing the three sub-objects:

(1) Step 1. Defining the two spherical segments SSI and $S S 2$, with function and mapping structure respectively, and describing them as sub-object $\mathrm{SO}_{1}$ and $\mathrm{SO}_{2}$ correspondingly.

Describe SSI based on spherical segment function (eq. (14)):

$[S S 1]=\left\{\right.$ "spherical segment", $\left\{\left\{P_{01}, 6.0,0.0,0.0,2.0\right\}\right.$, $\{\geqslant, \leqslant\}\}$, where $R$ (the radius the corresponding sphere) $=6.0 \mathrm{~m}, S$ (the distance between the spherical center to the spherical segment facet $)=2.0 \mathrm{~m}$; the spherical segment facet is horizontal and the building's up facet (in which the spherical segment facet lies on) is in east-west direction, thus $\alpha=0.0, \beta=0.0 ; P_{01}$ and $P_{02}$ is the coordinate of the two

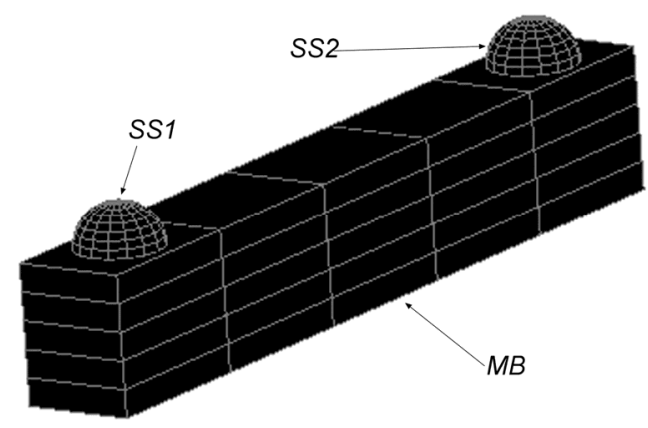

Figure 9 A building with two spherical segments. Main building $(M B)$, spherical segment (SS1, SS2). spherical centers respectively; operator $o p_{1}=$ " $\geqslant$ " and $o p_{2}=" \leqslant "$ denotes $F \geqslant S$ and $r \leqslant R$ (where $r=\sqrt{x^{2}+y^{2}+z^{2}}$ ) respectively.

Define sub-object $S O_{1}$ based on eq. (22d) (without RING):

$\left[\mathrm{SO}_{1}\right]=\{$ "function structure", \{"spherical segment", $\left.\left.\left.\left\{\left\{P_{01}, 6.0,0.0,0.0,2.0\right\},\{\geqslant, \leqslant\}\right\}\right\}\right\}\right\}$.

Define SS2 based on function mapping structure (eq. (21)) (using OFFSET, rather than ATTITUDE):

$[S S 2]=\left\{P_{02}, I N D E X\right\}$, where $P_{02}$ is the spherical center, i.e. OFFSET with respect to the global coordinate system; INDEX is the data pointer of sub-object $\mathrm{SO}_{1}$.

Describe sub-object $\mathrm{SO}_{2}$ based on eq. (22d) (without RING):

$\left[\mathrm{SO}_{2}\right]=\left\{\right.$ "function mapping structure", $\left.\left\{P_{02}, I N D E X\right\}\right\}$.

(2) Step 2. Define the main building $(M B)$ as sub-object $\mathrm{SO}_{3}$ using right prism.

Describe the $M B$ based on eq. (8):

$[M B]=\left\{\right.$ "right prism", $4,\left\{\left\{P 2 D_{1}, P 2 D_{2}, P 2 D_{3}, P 2 D_{4}\right\}, z_{0}\right.$, $h\}\}$, where "right prism" is the trip type of $D B$ structure, the right prism has 4 vertices $\left(P_{1}-P_{4}\right)$ on its bottom facet, $P 2 D_{1}-P 2 D_{4}$ is the corresponding $2 \mathrm{D}$ coordinate of the vertices, $z_{0}$ is the elevation of the bottom facet, and $h$ is the height of the right prism.

Define $\mathrm{SO}_{3}$ on the basis of eq. (22d) (without $\mathrm{RING}$ ):

$\left[\mathrm{SO}_{3}\right]=\left\{\right.$ "DB structure", \{ "right prism", 4, $\left\{P 2 D_{1}\right.$, $\left.\left.\left.P 2 D_{2}, P 2 D_{3}, P 2 D_{4}\right\}, z_{0}, h\right\}\right\}$, where structureType = "DB structure"; as a solid sub-object, $[S A]=\varphi$ (an empty set); since there are no RING(s), $n r$ and [RING] do not exist.

(3) Step 3. Synthesize the three sub-objects into sub-object $S O$ (it can also be expressed as an object).

Describe the structure data of $S O$ based on eq. (12b) (discrete object structure without $B O X$ ), and construct the sub-object model using eq. (22d) (without RING):

$[\mathrm{SO}]=\left\{\right.$ "discrete object structure", $\left\{3,\left\{\left[\mathrm{SO}_{1}\right],\left[\mathrm{SO}_{2}\right]\right.\right.$, $\left.\left.\left.\left[\mathrm{SO}_{3}\right]\right\}\right\}\right\}$, where structureType $=$ "discrete object structure".

\subsubsection{Example 3}

Modeling a complex building (Figure 10).

Figure 10(d) and (e) illustrates respectively the front view (oriented to the South) and the back view (oriented to the North) of a three-unit, eight-storey building, while Figure 10(c) shows the 2D RING of the building. By making use of index mapping structure based on the (sub-object) model ([SO $\left.O_{1}\right]$ ) of unit 1 (Figure $10(\mathrm{a})$ ), the (sub-object)

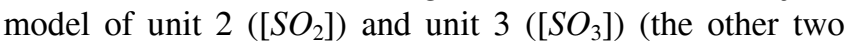
building units) can be achieved respectively. In addition, two other sub-objects $\left(\left[\mathrm{SO}_{4}\right],\left[\mathrm{SO}_{5}\right]\right)$ are used to represent the windows of the building in the east and west side respectively. The object model of the building is finally established by synthesizing these five sub-objects. The entire modeling process is detailed as follows:

(1) STEP 1. Construct the model of building unit 1. Figure 


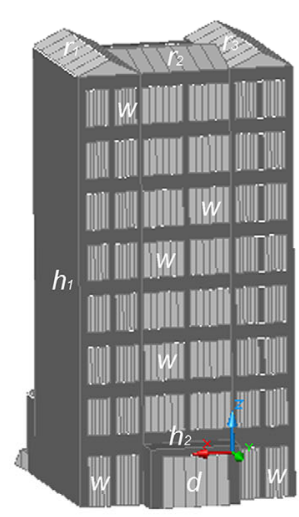

(a)

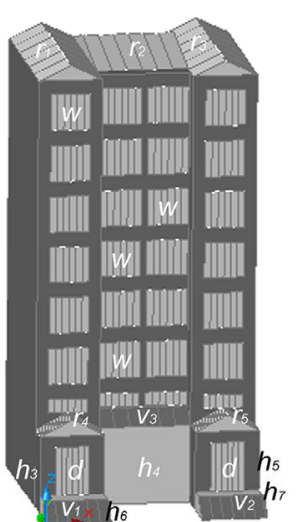

(b)

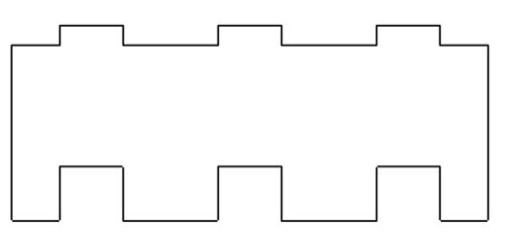

(c)

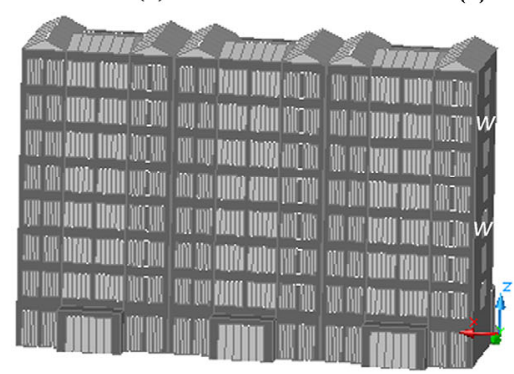

(d)

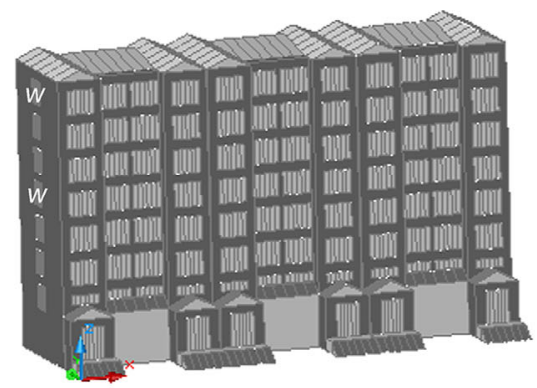

(e)

Figure 10 A complex building model. (a) Building unit (front view), (b) building unit (back view), (c) the RING of the building, (d) the building (front view), and (e) the building (back view).

$10 \mathrm{a}$ and $\mathrm{b}$ respectively demonstrates the front view and the back view of building unit 1 , where $w$ and $d$ denotes window and door respectively, $h$ is hexahedron, $v$ is wedge, $r$ is house ridge, $h_{1}$ is the main building of unit $1 ; r_{1}, r_{2}$ and $r_{3}$ refers to the three ridges respectively. In the front view (Figure 10a), $h_{2}$ is a hexahedron-shaped door house; there is a building door $(d)$ and four large windows $(w)$ in the first floor, and 6 windows on each of the other floors (floor 2-8). In the back view (Figure 10b), $h_{3}$ and $h_{5}$ are two hexahedron-shaped door houses, with two hexahedrons $\left(h_{6}\right.$ and $\left.h_{7}\right)$ below, two house ridges $\left(r_{4}\right.$ and $r_{5}$ ) on the top, and two wedge steps $\left(v_{1}\right.$ and $\left.v_{2}\right)$ in front of them; $h_{4}$ is a side house, with a wedge roof cover $\left(v_{3}\right)$ lying on the top of it; there are two windows on the second floor, and 4 windows on each of the floor 3-8. The building unit is described as a surface-solid composite sub-object [SO $\left.\mathrm{S}_{1}\right]$, including a solid sub-object $\left(\left[S O_{11}\right]\right)$ for all discrete bodies $(D B s)\left(h_{1-7}, r_{1-5}\right.$, $\left.v_{1-3}\right)$, two surface sub-objects in the front view for the windows and the doors in the first floor ([SO $\left.\left.\mathrm{S}_{12}\right]\right)$ and for the windows of the other floors $\left(\left[\mathrm{SO}_{13}\right]\right)$ respectively, and two surface sub-objects in the back view respectively for the doors in the first floor and the windows in the second floor ([SO $\left.\left.\mathrm{SO}_{14}\right]\right)$ and for the windows of the floor 3-8 ([SO $\left.\left.\mathrm{S}_{15}\right]\right)$.

Step 1-1. Describe the discrete bodies $(D B \mathrm{~s})$ of the solid sub-object ([SO $\left.\left.\mathrm{S}_{11}\right]\right)$ by representing $h_{1-7}\left(\left[H_{1-7}\right]\right), r_{1-5}\left(\left[R_{1-5}\right]\right)$ and $v_{1-3}\left(\left[V_{1-3}\right]\right)$ with right prism, ridge and wedge respectively, and construct the sub-object $\left(\left[S O_{11}\right]\right)$ with these 15 $D B$ s:
$\left[S O_{11}\right]=\left\{\right.$ "DB structure", $\left\{15,\left\{\left[H_{1}\right],\left[H_{2}\right],\left[H_{3}\right],\left[H_{4}\right]\right.\right.$, $\left[H_{5}\right],\left[H_{6}\right],\left[H_{7}\right],\left[R_{1}\right],\left[R_{2}\right],\left[R_{3}\right],\left[R_{4}\right],\left[R_{5}\right],\left[V_{1}\right],\left[V_{2}\right]$, $\left.\left.\left.\left[V_{3}\right]\right\}\right\}\right\}$.

Step 1-2. Describe the windows and doors in the front view and the back view respectively.

Based on MCT (tripType) (eq. (5)), represent the windows and the door in the first floor. The two windows are described as $\left[D C S_{11}\right]$ while the door as $\left[D C S_{12}\right]$. Hereinto, $\left[D C S_{11}\right]=\{$ "MCT", 2, 4, $[P]\}$, in which, the number of $D C S \mathrm{~s}$ and the vertices of each $D C S$ is 2 and 4 respectively, $[P]$ is the family set of vertices; similarly, there exists $\left[D C S_{12}\right]=\{" M C T ", 1,4,[P]\}$. The sub-object $\left(\left[S O_{12}\right]\right)$ is then built with the two DCSs based on eq. $(22 \mathrm{~d})$ : $\left[S O_{12}\right]=$ $\left\{\right.$ [DCS structure", $\left.\left\{2,\left\{\left[D C S_{11}\right],\left[D C S_{12}\right]\right\}\right\}\right\}$.

Describe the windows on floor 2-8 as surface sub-object $\left(\left[S_{13}\right]\right)$, based on eq. (15b): $[W D L]=\{$ "window or door Looping", (0.0, -1.0, 0.0), 126.0, 7, 3.6, \{“MCT", 6, 4, $[P]\}\}$, where the plane of windows is oriented to due south, thus $U N=(0.0,-1.0,0.0) ; h_{0}$ (elevation of the start floor $)=$ $126.0 \mathrm{~m}, n f$ (number of floors) $=7, n s$ (number of each floor's windows) $=6, n p$ (edges of a window $)=4,[P]$ is the family set of vertices of the windows in the second floor. Sub-object $\left(\left[\mathrm{SO}_{13}\right]\right)$ can be expressed as: $\left[\mathrm{SO}_{13}\right]=\{$ "function structure", $[W D L]\}$ (eq. (22d)).

Similarly, in the back view, the two doors in the first floor and four windows in the second floor are described as two DCSs respectively, and then defined as surface sub-object $\left(\left[\mathrm{SO}_{14}\right]\right)$; while the windows on floor 3-8 as sur- 
face sub-object ([SO $\left.\left.\mathrm{S}_{15}\right]\right)$ using eq. (15b).

Step 1-3: Based on eq. (12b), describe the structure data $\left(\left[D-S O_{1}\right]\right)$ of building unit 1 , and then define the corresponding sub-object $\left.\left[S O_{1}\right]: 1\right)\left[D-S O_{1}\right]=\left\{5,\left\{\left[S O_{11}\right],\left[S O_{12}\right]\right.\right.$, $\left.\left.\left[\mathrm{SO}_{13}\right],\left[\mathrm{SO}_{14}\right],\left[\mathrm{SO}_{15}\right]\right\}\right\}$, where $\left[\mathrm{SO}_{11-15}\right]$ refers to the above mentioned sub-objects; 2) describe the sub-object based on eq. (22d) (without $R I N G),\left[S O_{1}\right]=\{$ "discrete object structure", $\left.\left[D-S O_{1}\right]\right\}$.

(2) STEP 2. Respectively represent building units 2 and 3 as sub-object $\left(\left[\mathrm{SO}_{2}\right]\right)$ and $\left(\left[\mathrm{SO}_{3}\right]\right)$ using offset mapping structure.

Based on eq. (20), the structure data of unit 2 ([D-SO $]$ ) and unit $3\left(\left[D-S_{3}\right]\right)$ is respectively expressed as: $\left[D-S O_{2}\right]=\left\{\left(x_{2}, y_{2}, z_{2}\right), \quad I N D E X\right\}, \quad\left[D-S O_{3}\right]=\left\{\left(\begin{array}{lll}x_{3}, & y_{3}, & z_{3}\end{array}\right)\right.$, $I N D E X\}$; where $\left(x_{2}, y_{2}, z_{2}\right)$ and $\left(x_{3}, y_{3}, z_{3}\right)$ refers to the local coordinate system's origin of building units 2 and 3 respectively; INDEX is the data pointer of the unit 1's structure data (i.e. $\left.\left[D-S O_{1}\right]\right)$

Based on eq. (22d), $\left[\mathrm{SO}_{2}\right]$ and $\left[\mathrm{SO}_{3}\right]$ can be respectively expressed as: $\left[\mathrm{SO}_{2}\right]=\left\{\right.$ "offset mapping structure", $\left\{\left(x_{2}, y_{2}\right.\right.$, $\left.\left.\left.z_{2}\right), I N D E X\right\}\right\},\left[\mathrm{SO}_{3}\right]=\left\{\right.$ "offset mapping structure", $\left\{\left(x_{3}, y_{3}\right.\right.$, $\left.\left.\left.z_{3}\right), I N D E X\right\}\right\}$, where "offset mapping structure" is the structure type.

(3) STEP 3. Based on eq. (15b), describe the windows on the east and west side as sub-object $\left[\mathrm{SO}_{4}\right]$ and $\left[\mathrm{SO}_{5}\right]$ respectively: $\left[\mathrm{SO}_{4}\right]=\left\{\right.$ "window or door looping", $S N_{1}, h_{01}, n f, h$, $\left\{\right.$ \{ $\left.\left.M C T ", n s, n p,\left[P_{E}\right]\right\}\right\},\left[S O_{5}\right]=\{$ "window or door looping", $S N_{2}, h_{02}, n f, h,\left\{\right.$ "MCT", $\left.\left.n s, n p,\left[P_{W}\right]\right\}\right\}$, where $S N_{1}$ and $\mathrm{SN}_{2}$ refers to the plane's unit norm of the eastern and western side respectively; $\left[P_{E}\right]$ and $\left[P_{W}\right]$ is the vertex family set of the start floor's windows in the eastern and western side respectively, while $h_{01}$ and $h_{02}$ is the elevation of the windows respectively; $n f$ is the number of the floors, and $h$ is the height of each floor.

(5) STEP 4. Synthesize the model of the building.

Based on eq. (12b), describe the structure data $([D-O B J])$ of the building: $[D-O B J]=\{$ "discrete object structure", $\{5$, $\left.\left.\left\{\left[\mathrm{SO}_{1}\right],\left[\mathrm{SO}_{2}\right],\left[\mathrm{SO}_{3}\right],\left[\mathrm{SO}_{4}\right],\left[\mathrm{SO}_{5}\right]\right\}\right\}\right\}$, where "discrete object structure" is the structure type.

Based on eq. (22c), construct the building's object model $([O B J])$, in which one 2D RING is designed (Figure 10(c)): $[O B J]=\{\{$ object Number, "composite", BOX $\},\{[D-O B J], 1$, $[R I N G]\}$, where objectNumber is the sequential number of the object, "composite" is the shape type (shapeType), BOX is the regular hexahedron bounding box; $[R I N G]=\{n p$, $[P 2 D]\}$ ( $n p$, the number of vertices on the RING; $[P 2 D]$ is the family set of vertices on the RING).

\subsection{Data length comparison}

CityGML2.0 and IFC $2 \times 4$ are loosely formatted text, so for a fair data length comparison with UKRM model, both of the data has been handled as follows: all of the information irrelevant to geometry is removed first; the text format is then changed into a compacted structure in line with UKRM model: character constant as enumerated type (4 bytes), sequential or nominal indexing variable as data pointer type ( 4 bytes), integer value 4 , and double precision value 8 bytes. Meanwhile, BOX (the bounding box) in UKRM is omitted, considering the fact that it is just for the purpose of improving the efficiency of data query and analysis, rather than a necessity for 3D modeling. Table 1 lists the data amount of different methods for the three modeling examples respectively, which suggests that the data length of UKRM is less than either of other two data models. Note: the level of details in the examples is equivalent to LOD3, thereby only calculating the geometric data size of LOD3 of CityGML.

\section{Discussion and conclusions}

Data model. To represent the complicated and diversified three-dimensional world efficiently and conveniently, and satisfy the application requirements of diverse GIS, ubiquitous knowledgeable representation model (UKRM) is proposed here, which consists of three structure types: discrete, function and mapping structure, and each of which contains several sub-classes of structures. A new structure (i.e. basic element) can be easily integrated by simply presenting its structure module and its ID number. Thanks to such a modular design strategy, UKRM possesses the desirable properties: being back compatible, extensible and maintainable (Bass et al., 2003). These heterogeneous structures are made uniform with structure type (structureType), thus realizing the integration of multi-expression. Any of the structure types or their combination can be used to describe a spatial object, and a suitable modeling approach can be chosen by a GIS user according to the application demands and/or his (or her) own preference. For example, example 1 adopts different DCS structures, and example 2 utilizes discrete body $(D B)$, function and mapping structure jointly; while $D C S, D B$, function structure and mapping structure are combined simultaneously in example 3 . These examples demonstrate that, whether for regular or irregular geometries, the modeling efficiency of UKRM is superior to other commonly used data models: its data volume is less than that of IFC, and much less than that of CityGML, up to several dozen times or even more (Table 1). It should be pointed out that the adoption of multiple expressions exerts no impact on the usage of semantics; instead, the assign-

Table 1 Data amount comparison of different modeling approaches

\begin{tabular}{|c|c|c|c|c|}
\hline \multirow{2}{*}{ Data model } & \multicolumn{4}{|c|}{ Data lengths of different objects (Byte) } \\
\hline & \multicolumn{2}{|c|}{ Example 1} & Example 2 & Example 3 \\
\hline UKRM model & $\begin{array}{c}\text { Topology } \\
488\end{array}$ & $\begin{array}{c}\text { Non-topology } \\
752\end{array}$ & 192 & 4172 \\
\hline CityGML2.0 & & 2304 & 31720 & 51336 \\
\hline IFC $2 \times 4$ & 736 & & 536 & 10168 \\
\hline
\end{tabular}


ment of semantic attributes can be carried out at different hierarchical levels: the level of object, sub-object and structure (i.e. basic element) respectively. Based on UKRM model, a new open and knowledgeable 3D GIS data standard can be further established. GIS users can just reuse the existing structures or perform maintenance on new structures' extension, rather than get stuck in the current issue of "one project one discussion" in 3D GIS modeling.

Discrete structure. The proposed three powerful structure types provide efficiencies in data storage and data representation. Each of structure types has its own merits, and all of them fully depict the regular geometric characteristics from different aspects. Hereinto, discrete unit $(D U)$ structure (DCS or $D B$ ), the major form of discrete structure, not only comprises the discrete data (e.g. vertices) of object (or sub-object), but includes geometric parameters. For example, $n s$ (number of facets) and $n s p$ (number of vertices in each facet) are used in $M C T$ (eq. (5)), while geometric parameters are also found in house ridge, right prism, etc. Of course, defining a basic discrete element without parameters is permissible too (i.e. equivalent to traditional modeling methods). For DCS structure, because the common geometric properties of facets are described collectively, the corresponding modeling efficiency is therefore enhanced. Taking triangle fan as an example, the relationship between facets and vertices of a triangle fan is about $1: 1$ (eq. (2)), whereas that in traditional GS data models is at least $1: 3$. And it is demonstrated by example 1 that, the number of vertices stored for non-topological structure is no more than two times of the total vertices in the ore body. Undoubtedly, the data redundancy of boundary representation (B-rep) has greatly been reduced by DCS structure. With a variety of expressions (e.g. triangle fan, triangle strip, double-ruled surface, etc.), in the form of topology or non-topology, DCS can be widely used for the description of the surface object or the solid object enclosed by a regular or irregular surface, e.g. geological body.

Compared with $D C S, D B$ structure is more suitable for describing regularly shaped three-dimensional solids (e.g. buildings). By so far, plenty of geometric knowledge about solid figures has been mastered by human beings, and used widely in industries like engineering, architecture, etc. Such knowledge can be utilized in describing $D B$ structure. In fact, models such as TEN (Pilouk, 1996), GTP (Wu, 2004), etc, belong to $D B$ structure. Different from the polyhedron commonly used in current GIS (e.g. Arens et al., 2005), each of the polyhedrons defined by $D B$ structure has a unique shape that is familiar to human beings. As a consequence, all of a solid primitive's lower dimensional elements are unnecessary to be explicitly presented, thus leading to a significant reduction of data redundancy. For instance, there are no line and facet elements in the model right prism (or ridge), while vertices might be just described with their $2 \mathrm{D}$ coordinates, and some vertices may even be omitted. It should be especially pointed out that, with the help of $D B$ structure, generation of 3D building models from $2 \mathrm{D}$ architectural plans could be realized. Once a $D B$ is defined, its three-dimensional shape (i.e. shape semantics) is then understood by human beings, meaning that the basic primitives defined by $D B$ structure are consistent with human habits of thinking and observing. Of course, the basic units defined by DCS structure can be triangle or polygon (eq. (6)), while $D B$ structure can use tetrahedron in order to express an irregular solid. That is, discrete unit $(D U)(D C S$ or $D B$ ) is compatible with current GIS data models, ensuring its completeness and adaptability of spatial data description. But make no mistake: $D C S$ and $D B$ are not in conflict, without recursive references and/or mutual references.

Function structure. Compared to discrete structure, function structure is more flexible and efficient, whose parameters can be general variables, parameters, operators, $D U$ (DCS or $D B$ ), and pointer of indexing data (i.e. function mapping structure), etc. Different from numerical computation, the function defined by function structure is an object (or sub-object), rather than a numerical value. It is well known that traditional GIS models represent a higher dimensional element with a lower dimensional one step-bystep. This convention, however, will be modified, because of the incorporation of function structure, in which a spatial object (or sub-object) might be directly expressed without lower dimensional data, for instance, there are no vertices, lines and facets in a sphere in function structure. At the same time, functional representation has a peculiar characteristic: scale invariance (the data length of a function at different scales remains the same). In consequence, both the accuracy and the efficiency of data representation have been boosted. Moreover, the adoption of function structure is conducive to the seamless data conversion (i.e. data integration) between GIS and other 3D software. Due to the absence of vertices, the inner/outer property of the surface could not be determined as usual: based on the clockwise (or counter-clockwise) order of vertices. So [SA] (family set of surface attributes) is presented in UKRM model (eq. (22)). For most cases, surface stands for outer surface, for example, when taking a cavern as a solid object, its surface is also an out surface.

Mapping structure. With the basic primitives (i.e. building blocks) defined by $D C S, D B$ and function structure, it becomes easy and convenient to model a spatial object, which is carried out by simply piling up the building blocks (see examples 1-3). While mapping structure reuses these building blocks or the spatial data constructed by them through direct data reference or through ATTITUDE and/or OFFSET transformation. For instance, function is reused in example 2, while the data of a whole building unit 1 is referenced through OFFSET transformation in example 3, in which the spatial data is not generated and stored iteratively. Drawing experience from construction and engineering industry, mapping structure approximates engineering drawing and computer software language; it reduces the amount of data storage, simplifies the process of data production, 
decreases data errors of human input, and enables construction materials to be reused. Any shape type of a spatial object (point, surface, solid, composite) can serve as an elementary object for reference, which can be applied at any scale, e.g. the same shaped buildings in a housing estate; the same structured building units, floors and rooms within a building, as well as the identical balconies outside of the building. The introduction of mapping structure hence brings light to make breakthrough for 3D GIS modeling at multi-scale (inner and outside of a building) and about massive objects. In 2D GIS, geometric parameters like orientation play an important role, while in 3D GIS, the description of ATTITUDE is even more universal and useful, e.g. the expression of buildings at different scales. Moreover, this structure type would be advantageous in 3D WebGIS applications too, thanks to the small amount of data storage and network data transmission.

It is important to note that, in spatial-temporal data structure, a moving object's local coordinate system itself is a moving framework (Chen and Chen, 1983). Any static object can be regarded as the instant state of a moving object, thus ATTITUDE can be viewed as the instant states of the moving object at different time. Therefore, the incorporation of mapping structure can not only describe static objects, but set up the extension interface from static to dynamic GIS. For the representation and analysis of the trajectory of a dynamic object (e.g. satellite), utilization of function structure is inevitable to calculate and predict the object's trajectory, time, etc.; whereas for the description of the moving object's attitudes, mapping structure is a necessity. From this respective, we can say that the incorporation of function and mapping structure is the inevitable consequence of GIS development.

3D spatial attributes. The structure of attributes in UKRM model is organized as follows: class (or file) attributes, stored in the file header, belong to the thematic attribute of the entire class; object attributes, stored in data header of an object (eq. (22)), equal to file attributes in default, are assigned to the whole object, including all of its sub-objects, discrete unit $(D U)$ (DCS or $D B)$; similarly, sub-object or $D U(D C S$ or $D B)$ attributes are applied only to the corresponding sub-object or the $D U(D C S$ or $D B)$. Moreover, each $D B$ 's facets and vertices are numbered, allowing spatial attributes (e.g. texture, vertex color, lighting and material, etc.) to be assigned to a particular facet or vertex (or to certain facets or vertices). By doing so, a "class $\rightarrow$ object $\rightarrow$ sub-object $\rightarrow D B \rightarrow$ facet $\rightarrow$ vertex" multiclass and multi-level attribute management is performed.

Data storage and management of UKRM model. UKRM model supports both file and database data storage. The structure of the file data storage is: file header, \{data header, graph data\} (eq. (22)), where the later item (those within the brace) is the data set of spatial objects while the former item is the information of the entire graph layer. In the case of the database data storage, the structure data is stored as a BLOB data type in a column, followed by other attributes (including those defined by users) in other data fields. As for a complex scene, a multi-layer and multi-class data storage and management strategy can be employed, e.g. building layer, terrain layer, etc.

Data validation and verification, uniqueness and completeness. Data verification: instead of using Euler formula (a traditional method), the formulas defined by various trip types, as well as those given by function and mapping structure are employed here (Zhang et al., 2004). For instance, the relationship among tripType, $n p$ and $[P]$ can be used for $D C S$ (explicit trip types) data verification, while the data of discrete body $(D B)$ structures can be validated according to the formula defined in different trip types. Uniqueness: the accuracy difference between discrete structure and function structure is within the permissible scope of GIS. Hence, whether described by discrete structure or function structure, the geometric position of a spatial object is unique. As for mapping structure, it is a rigid body transformation (ATTITUDE, OFFSET) of discrete or function structure, i.e. it belongs to one of the two structure types, so the spatial position expressed by mapping structure is unique too (see example 3). Completeness: UKRM model is able to represent the geometry, semantics and topology of any spatial entity efficiently. Hereinto, topology is described inexplicitly: for discrete structure, after traversing the intersection of all point, line, surface and solid elements between two geometries, where and how the geometries intersect can be computed (Zhang and Zhang, 2010); for function structure, if it is a functional geometry enclosed by discrete point, line and surface elements, topological relations are calculated as above, while if continuous curved surface(s) exist, the intersection relationship can be computed by mathematical analysis; for mapping structure, the calculation of topology is then changed as one of the above cases. As a matter of fact, topological relations do not vary from the types of geometric expressions.

2D RING. RING is to describe the 2D boundary of the projection of a spatial object (or sub-object), e.g. the boundary of ore body, geological body, ground object, and building, etc., and it plays an especially important role in relation to the terrain surface. RING is generated by drawing the boundary of the spatial object's projection on the terrain surface, and it is stored in the same data file with the 3D graphics data. That is, without taking up an extra storage space, the 2D and 3D data are stored as one data set. Thus the definition of RING sets up the theoretical foundation of $2 \mathrm{D} / 3 \mathrm{D}$ data integration. In comparison with the $3 \mathrm{D}$ ring of CityGML, i.e. terrain intersection curve (TIC), the RING proposed in this study has wider applicability: it is even applicable to any above ground or underground objects. Meanwhile, the TIC can be obtained through geometric computation, thereby avoiding manual efforts in data generation and storage.

Spatial data query and analysis. UKRM model improves the granularity of data representation, and reduces the related aggregation operations; besides, a tree-like storage 
data structure is adopted. This would be conducive to GIS's ability in data management, data query and analysis, as proved by Zhang and Zhang (2010) that the efficiency of geometric intersection can be improved by a tree-like structure of "surface-sub-surface-facet". What's more, it is also for the purpose of data query and analysis to assign $B O X$ to an object (eq. (21)) and to each of those sub-objects with a large number of vertices (eq. (12a)). It should point out that the knowledgeable representation of basic units makes it is possible to perform shape-semantics based data query and analysis, to answer questions like "how many buildings with spherical segments in XX city (Figure 9); and where are they located?". This sort of spatial queries is especially beneficial to spatial location and analysis. Moreover, thanks to the properties of an object-oriented data model, spatial data at object (or sub-object) level in UKRM model is independent from each other. This would be suitable for the development and application of modern computational environments (e.g. high performance computation, cloud-computing). But due to the length limitation, detailed researches on GIS function and multi-purpose (-user) application will be given in the future.

In short, the existing discrete modeling approaches, including discrete curved surface $(D C S)$, discrete body $(D B)$, etc., are extended and integrated in this study, where parameters could be used in particular; two representation types, namely function structure and mapping structure are newly put forward; the three structure types are integrated under a flexible and extensible modeling framework. By doing so, multiple expressions are deepened and integrated, including boundary representation (B-rep), construction solid geometry (CSG), parametric/functional representation, mapping structure, etc., data is reused, 2D/3D data integration is realized, and multiple modeling choices are provided for GIS users. A spatial object can now be described with any of the structure types or their combinations, so that the diverse regular geometric properties of the spatial object can be captured. Data redundancy is thus reduced, and data modeling efficiency is improved. Besides, the spatial data can be stored either in a data file, or in a database having binary large object (BLOB), which brings the database into full play in spatial data management. The complicated and diversified three-dimensional world can therefore be efficiently and flexibly represented and analyzed, and GIS application demands of multi-purpose (-user) are satisfied. The theoretical foundation for the development of knowledgeable GIS is hence set up.

Acknowledgements The authors are overwhelmed with gratitude to the three anonymous reviewers for their constructive and valuable comments and suggestions. We are also very appreciative to the paper review of the earlier version of this paper by professor May Yuan (the University of Oklahoma), and to the language revision by Mr. Rahman Gul. This research was supported by the National Natural Science Foundation of China (Grant No. 41271196), and the Key Project of the 12th Five-year Plan, Chinese Academy of Sciences (Grant No. KZZD-EW-07-02-003).

\section{References}

Arens C, Stoter J, Van Oosterom P. 2005. Modeling 3D spatial objects in a Geo-DBMS using a 3D primitive. Comput Geosci, 31: 165-177

Bass L, Clements P, Kazman R. 2003. Software Architecture in Practice. Boston: Addison-Wesley Longman Publishing. 49

Berlo L V, Laat R D. 2011. Integration of BIM and GIS: The Development of the CityGML GeoBIM Extension. Inter 3D GeoInfo Conf, 5: 197-210

Breunig M, Zlatanova S. 2011. 3D Geo-database research: Retrospective and future directions. Comput Geosci, 37:791-803

Chen S S, Chen W H. 1983. Lectures on Differential Geometry (in Chinese). Beijing: Peking University Press. 178

Coors V. 2003. 3D GIS in networking environments. Comput Environ Urban, 27: 345-357

Gong J Y, Cheng P G, Wang Y D. 2004. Three-dimensional modeling and application in geological exploration engineering. Comput Geosci, 30: 391-404

Goodchild M F. 2009. Geographic information systems and science: Today and tomorrow. Ann GIS, 15: 3-9

Gröger G, Kolbe T H, Nagel C, Häfele K H. 2012. OGC City Geography Markup Language (CityGML) Encoding Standard Version 2.0. http://www.opengis.net/spec/citygml/2.0

Herring J. 2001. The OpenGIS Abstract Specification, Topic 1: Feature Geometry (ISO 19107 Spatial Schema) Version 5

Kuttig D. 2003. Potential and limits of functional modeling in the CAD process. Res Eng Des, 5: 40-48

Li Q Q, Li D R. 1998. Research on the conceptual frame of the integration of 3D spatial data model (in Chinese). Acta Geod Cartograph Sin, 27: 325-330

Lv G N, Yuan L W, Yu Z Y. 2013. Challenges to development and socialization of GIS technology (in Chinese). Geo-Inform Sci, 15: 483-490

Mallet J L. 2002. Geomodeling. New York: Oxford University Press. 624

Molenaar M. 1991. Formal data structures, object dynamics and consistency rules. In: Ebner H, ed. Digital Photogrammetric Systems. Karlsruhe: Wichmann Verlag. 262-273

Pilouk M. 1996. Integrated Modeling for 3D GIS. Doctoral Dissertation. Netherlands: Wageningen Agricultural University and ITC. 58

Reitsma F. 2013. Revisiting the 'Is GIScience a science?' Debate (or Quite Possibly Scientific Gerrymandering). Int J Geogr Inf Sci, 27: 211-221

Shi W Z, Yang B S, Li Q Q. 2003. An object-oriented data model for complex objects in three-dimensional geographic information systems. Int $\mathrm{J}$ Geogr Inf Sci, 17: 411-430

Wu L X. 2004. Topological relations embodied in a generalized tri-prism (GTP) model for a 3D geosciences modeling system. Comput Geosci, 30: 405-418

Xiao L B, Zhong E S, Liu J Y, Song G F. 2001. A discussion on basic problems of 3D GIS (in Chinese). J Image Graph, 6: 30-36

Yuan L W, Yu Z Y, Lou W, Zhou L C, Lü G N. 2011. A 3D GIS spatial data model based on conformal geometric algebra. Sci China Earth Sci, 54: 101-112

Zhang S Q, Zhang J Y. 2010. Theoretical analytics of stereographic projection on 3D-objects' intersection predicate. Int J Geogr Inf Sci, 24: 25-46

Zhang S Q, Zhang J Y, Zhang B. 2004. Deduction and application of generalized Euler Formula in topological relation of geographic information system. Sci China Ser D-Earth Sci, 47: 749-759

Zhou L C, Lü G N, Sheng Y H, Xu H B, Wang H X. 2008. A 3D GIS's spatial data model based on Cell Complex. In: The International Archives of the Photogrammetry. Remote Sensing Spat Information Sci, 21: 905-908

Zhu Q, Hu M Y. 2008. Semantics-based LOD models of 3D house property (in Chinese). Acta Geod Cartograph Sin, 37: 514-520

Zhu Q. 2014. Full three-dimensional GIS and its key roles in smart city (in Chinese). Geo-Inform Sci, 16: 151-157

Zlatanova S, Tempfli K. 2000. Modeling for 3D GIS: Spatial analysis and visualization through the web. In: Proceedings of the XIX Congress of ISPRS. 1257-1264

Zlatanova S, Rahman A A, Shi W Z. 2004. Topological models and frameworks for 3D spatial objects. Comput Geosci, 30: 419-428 